Pesq. Vet. Bras. 30(4):358-362, abril 2010

\title{
Atividade antioxidante de macrófagos alveolares em ratos endotoxêmicos ${ }^{1}$
}

\author{
Juliana Félix de Melo ${ }^{2}$, Érika M. Correia de Macedo², Rebecca P. Paes \\ Silva ${ }^{2}$, Marcelo Tavares Viana ${ }^{3}$, Wylla T. Ferreira e Silva ${ }^{3}$ e Célia M.M. \\ Barbosa de Castro ${ }^{2}$
}

\begin{abstract}
Melo J.F, Macedo E.M.C., Paes Silva R.P., Viana M.T., Ferreira e Silva W.T. \& Castro C.M.M.B. 2010. [Anti-oxidative activity of alveolar macrophages in endotoxemic rats.] Atividade antioxidante de macrófagos alveolares em ratos endotoxêmicos. Pesquisa Veterinária Brasileira 30(4):358-362. Laboratório de Imunopatologia Keizo Asami, Setor de Microbiologia, Universidade Federal de Pernambuco, Recife, PE 50670-420, Brazil. E-mail: julemelo@hotmail.com

The effects of endotoxemia on the antioxidant activity in alveolar macrophages of Wistar rats were evaluated. Twenty-four male rats, 90-120 days of age, were separated into 2 groups: control and endotoxemic. To the endotoxemic animals was administered, intraperitoneally, a lipopolyssaccaride at dosage of $1 \mathrm{mg} / \mathrm{kg}$ body weight. Twenty-four hours after this procedure, blood was collected for total and differential leukocytes counts. In addition, bronchoalveolar lavage was collected for total and differential leukocyte counting. From this lavage macrophages were isolated for the dosage of superoxide and superoxide dismutase. The endotoxemia increased the total leukocyte counts and the number of neutrophils in the peripheral blood and bronchoalveolar lavage of the rats. There was an increased superoxide production without changing the superoxide dismutase. Our findings indicate that endotoxemia induces lung inflammatory response. However, it does not alter the antioxidant activity in adult rats. This fact not only enhances host response against infectious agents, but might also contribute to the pathogenesis of pulmonary injury.
\end{abstract}

INDEX TERMS: Antioxidant activity, oxidant activity, endotoxemia, cellular recruitment, macrophage.

RESUMO.- Avaliou-se o efeito da endotoxemia sobre a atividade antioxidante de macrófagos alveolares em ratos da linhagem Wistar. Foram utilizados 24 ratos machos, com idade entre 90 e 120 dias, os quais foram divididos em dois grupos: controle e endotoxêmico. O grupo endotoxêmico foi submetido à injeção intraperitonial de lipopolissacarídio na dose de $1 \mathrm{mg} / \mathrm{kg}$ de peso corporal.

\footnotetext{
${ }^{1}$ Recebido em 24 de abril de 2008.

Aceito para publicação em 18 de dezembro de 2009.

Este estudo recebeu apoio financeiro do CNPq.

${ }^{2}$ Laboratório de Imunopatologia Keizo Asami (LIKA), Setor de Microbiologia, Universidade Federal de Pernambuco (UFPE), Cidade Universitária, Recife, PE 50670-420, Brasil. *Autor para correspondência: julemelo@hotmail.com

${ }^{3}$ Laboratório de Fisiologia do Departamento de Nutrição, Universidade Federal de Pernambuco (UFPE), Cidade Universitária, Recife, PE 50670-901.
}

Após $24 \mathrm{~h}$, coletou-se sangue para contagem total e diferencial de leucócitos; lavado broncoalveolar para contagem total e diferencial dos leucócitos e, a partir de macrófagos isolados deste lavado, foram realizadas as dosagens de superóxido e superóxido dismutase. A endotoxemia aumentou a contagem total de leucócitos e o número de neutrófilos no sangue periférico, no lavado broncoalveolar, e aumentou a produção de superóxido sem modificar a produção da superóxido dismutase. Esses resultados sugerem que a endotoxemia induz a uma resposta inflamatória no pulmão. Contudo, não altera a atividade antioxidante em ratos adultos. Tal fato potencializa a resposta contra agentes infecciosos pelo hospedeiro, mas também pode contribuir na patogênese de injúria pulmonar.

TERMOS DE INDEXAÇÃO: Atividade antioxidante, atividade oxidante, endotoxemia, recrutamento celular, macrófago. 


\section{INTRODUÇÃO}

Em linhas gerais, os eventos que decorrem da sepse e do choque séptico em modelos animais são causados por endotoxinas ou lipopolissacarídios (LPS) produzidos por bacilos Gram negativos. Essas substâncias se localizam na parte externa da parede celular bacteriana e são liberadas a partir da sua replicação e/ou morte (De Castro et al. 1997). A endotoxemia aguda, caracterizada por altos níveis de endotoxina no sangue, causa reação inflamatória com injúria endotelial, hipotensão, falência múltipla dos órgãos e morte (Hewett \& Roth 1993, Sunil et al. 2002).

Dentre os órgãos envolvidos no choque séptico, o pulmão é um dos mais sensíveis aos efeitos das endotoxinas e o primeiro a ser atingido (D'Acampora et al. 2001). As complicações pulmonares, incluindo edema pulmonar e falência respiratória, são as maiores causas de morbimortalidade durante a endotoxemia (Wang et al. 1994).

A ativação dos macrófagos por endotoxinas induz a produção intracelular dos ânions superóxido $\left(\mathrm{O}_{2}^{-}\right)$, peróxido de hidrogênio $\left(\mathrm{H}_{2} \mathrm{O}_{2}\right)$ e outros potentes produtos microbicidas que são responsáveis pela destruição dos microrganismos fagocitados (Meyer et al. 1994, Amersfoort et al. 2003).

Em contrapartida, os compostos antioxidantes protegem os sistemas biológicos contra os efeitos potencialmente danosos de reações das espécies reativas de oxigênio com diversos alvos celulares (Mafra et al. 1999).

A administração parenteral de LPS mimetiza a reação generalizada de defesa do organismo às infecções, como febre, anorexia e indução das proteínas de fase aguda no fígado (Dinarello 1996, Plata-Salamán 1996). Ademais, induz a migração de leucócitos sanguíneos para o sítio inflamatório/infeccioso. Assim, o LPS funciona como um adequado modelo para o estudo da manifestação de alterações fisiológicas durante infecções bacterianas.

Considerando que o pulmão é um dos órgãos mais sensíveis aos efeitos das endotoxinas, torna-se importante a realização de trabalhos experimentais no intuito de investigar as funções dos macrófagos alveolares nesta condição. Apesar de já existirem muitos estudos sobre o recrutamento celular e a atividade oxidante em ratos tratados com LPS, ainda são escassos os conhecimentos acerca do papel do sistema de defesa antioxidante, especialmente da superóxido dismutase, em animais endotoxêmicos. No presente estudo, pretende-se avaliar o efeito da endotoxemia sobre a atividade antioxidante de macrófagos alveolares em ratos Wistar.

\section{MATERIAL E MÉTODOS}

O protocolo deste estudo foi previamente aprovado pela Comissão de Ética em Experimentação Animal do Centro de Ciências Biológicas da Universidade Federal de Pernambuco (CEEA-UFPE) (Protocolo 23/05, aprovado em 9.6.2005).

Animais e administração de lipopolissaccarídeo (LPS). Foram utilizados 24 ratos machos Wistar (90-120 dias), provenientes da colônia do Biotério do Departamento de Nutrição, Universidade Federal de Pernambuco (UFPE). Os animais foram mantidos em temperatura controlada $\left(22 \pm 1^{\circ} \mathrm{C}\right)$, com ciclo fotoperiódico de claro-escuro de 12-12 h e livre acesso à água e a ração (Labina-Purina do Brasil). Os animais foram divididos em dois grupos: Controle (C) e Endotoxêmico (CLPS). O grupo CLPS foi submetido à injeção intraperitonial (i.p.) de LPS (sorotipo de Escherichia coli; 055:B5, Sigma) na dose de $1 \mathrm{mg} / \mathrm{kg}$ de peso corporal, preparado com $\mathrm{NaCl}$ a $0,9 \%$.

O grupo $\mathrm{C}$ recebeu, pela mesma via, $\mathrm{NaCl}$ a $0,9 \%$ em igual proporção.Vinte e quatro horas após a administração do LPS (De Castro et al. 1995), coletaram-se sangue e lavado broncoalveolar (LBA) de cada animal para estudo.

Análise das células do sangue periférico. Coletaram-se alíquotas de sangue $(0,5 \mathrm{~mL})$ da veia caudal dos animais, após anestesia, para a contagem das células. Utilizou-se a solução de TURK para a contagem total. Os leucócitos foram contados em Câmara de Neubauer com auxílio do microscópio óptico, com objetiva de 40x. Para a contagem diferencial utilizou-se a técnica de estiraço sanguíneo, e as lâminas foram coradas com o kit Panótico Rápido, LB-Laborclin Ltda. Após preparação, foram examinadas ao microscópio óptico com objetiva de 100x sob imersão. Os diferentes elementos foram contabilizados pelo contador eletrônico da marca Kacil com teclas correspondentes a cada tipo de célula. A partir dos dados obtidos foram calculados os valores absolutos e relativos para cada tipo de célula.

Lavado broncoalveolar (LBA). O lavado broncoalveolar (LBA) foi obtido de acordo com a técnica desenvolvida por De Castro et al. 1995. Os animais foram anestesiados com cloralose-uretana (0,5 e 12,5\% respectivamente) na proporção de $8 \mathrm{~mL} / \mathrm{kg}$ i.p. O LBA foi coletado com a injeção de salina a $0,9 \%$ através de uma cânula plástica inserida na traquéia. Várias alíquotas de $3 \mathrm{~mL}$ foram injetadas e coletadas em tubos cônicos de polipropileno de $50 \mathrm{~mL}$ (Falcon, Sigma). Recuperou-se aproximadamente $30 \mathrm{~mL}$ de LBA para cada animal.

Análise das células do lavado broncoalveolar. A contagem total das células foi realizada a partir de uma amostra do LBA, diluído de 1:10, em corante azul de trypan. A contagem diferencial foi realizada a partir de lâminas citocentrifugadas a 800 rpm/10min (Cytopro $^{\mathrm{TM}_{-}}$-Cytocentrifuge Wescor), coradas com o kit Panótico Rápido e lidas ao microscópio óptico com objetiva de 100x sob imersão. A partir dos dados obtidos foram calculados os valores absolutos e relativos para cada tipo de célula.

Cultura de macrófagos alveolares (MA). Centrifugou-se as amostras do LBA recolhido, a 1500rpm durante $15 \mathrm{~min}$. O precipitado que corresponde às células foi ressuspendido em RPMI 1640 (Gibco-Invitrogen Corporation) contendo 3\% de soro fetal bovino (Gibco-Invitrogen Corporation) e antibióticos (penicilina $100 \mathrm{U} / \mathrm{mL}$ e estreptomicina $100 \mu \mathrm{g} / \mathrm{mL}$ ). As células foram transferidas para placas de cultura de $35 \mathrm{~mm}$ de diâmetro (6 poços, Falcon), onde foram dispensados $2 \mathrm{~mL}$ da suspensão em uma proporção de $10^{6}$ células $/ \mathrm{mL}$ de RPMI 1640 em cada poço. Após $1 \mathrm{~h}$ na incubadora a $37^{\circ} \mathrm{C}$ e $5 \% \mathrm{CO}_{2}$, desprezou-se o sobrenadante com as células não-aderentes e adicionou-se $2 \mathrm{~mL}$ de meio RPMI, deixando-se as placas por mais $1 \mathrm{~h}$ em incubadora para estabilização das células.

Análise da produção de superóxido $\left(\mathrm{O}_{2}^{-}\right) \cdot \mathrm{O} \mathrm{O}_{2}^{-}$foi induzido pela adição de acetato miristato de forbol/PMÂ (Sigma) em solução de Hanks (HBSS, Gibco-Invitrogen Corporation), na concentração de $2 \mu \mathrm{g} / \mathrm{mL}$. Foram preparados 2 sistemas de análise descontínua com avaliação a cada 1 hora, por 2 horas. A especificidade do ensaio foi garantida pela adição de superóxido dismutase (SOD) de eritrócitos bovinos contendo $3000 U / \mathrm{mg}$ de proteína em solução final de $3 \mathrm{mg} / \mathrm{mL}$ em água destilada (Sigma) (De Castro et al. 2000). 
Para o preparo destes sistemas foram utilizados macrófagos em cultura ( $2 \times 10^{6}$ células por $2 \mathrm{~mL}$ de RPMI 1640$)$, com ou sem a presença de SOD. Os sistemas foram mantidos em incubadora a $37^{\circ} \mathrm{C}$, sob atmosfera úmida, $5 \%$ de $\mathrm{CO}_{2}$, por $10 \mathrm{~min}$ (ativação da SOD). O ferrocitocromo C tipo IV (de mitocôndria de coração de cavalo, $30 \mathrm{mg} / \mathrm{mL}$ em HBSS, $2.4 \times 10^{-3} \mathrm{M}$ (Sigma) foi adicionado às células para quantificar a formação de $\mathrm{O}_{2}{ }^{-}$ através da redução do ferrocitocromo $\mathrm{C}$. Amostras de $700 \mu \mathrm{\mu l}$ foram retiradas de cada sistema. A primeira alíquota recolhida correspondia ao tempo "zero" de cada sistema e as amostras subseqüentes foram coletadas em intervalos de 1 hora e 2 horas. Medidas espectrofotométricas foram realizadas a $550 \mathrm{~nm}$ para determinar o grau de redução do ferrocitocromo $\mathrm{C}$ dos sobrenadantes. A curva de $\mathrm{O}_{2}{ }^{-}$foi obtida pela conversão dos valores de absorbância para nanomoles de $\mathrm{O}_{2}-1$ min usando-se a fórmula: $[O]=205,49 \times$ valor da absorbância $\times$ volume da amostra coletada (Johnston 1984).

Atividade da enzima superóxido dismutase (SOD). A atividade da SOD foi determinada pelo preparo de 4 sistemas com avaliação nos tempos de 0,5,10 e 15min. Para o preparo destes sistemas, foram utilizados macrófagos em cultura $\left(2 \times 10^{6}\right.$ células por $2 \mathrm{~mL}$ de RPMl 1640), solução tampão fosfato ( $50 \mathrm{mM}$, pH 7,8), citocromo C (Sigma, 0,3mM - 4 $\mu \mathrm{cg} / \mathrm{mL}$ em solução tampão), xantina oxidase (XOD) (Sigma, $25 \mathrm{U}$ - 56mU/mL em solução tampão) e SOD (Sigma, 3000U/mg de proteína em solução final de $3 \mathrm{mg} / \mathrm{mL}$ em água destilada). Aos 4 sistemas foram adicionados solução tampão fosfato, citocromo $\mathrm{C}$ e hipoxantina. Ao $2^{\circ}$ sistema, além desses reagentes, também foi adicionada a XOD. Os Sistemas 3 e 4 foram acrescidos de todos os reagentes, sendo que a SOD em quantidades diferentes (Sistema 3, SOD 1,5mg/mL; Sistema 4, SOD $3 \mathrm{mg} / \mathrm{mL}$ ). Amostras de $700 \mu \mathrm{l}$ foram retiradas de cada sistema nos tempos determinados $\left(a 5^{\circ} \mathrm{C}\right)$, sendo efetuadas as leituras das absorbâncias em espectrofotômetro a $550 \mathrm{~nm}$. Para os resultados finais, as proteínas foram determinadas pelo método de Lowry (Lowry et al. 1951) e os dados expressos em UI de SOD por mg de proteína.

Análise estatística. $\mathrm{Na}$ comparação entre os grupos, utilizou-se o teste t de Student para os dados paramétricos e o teste de Mann-Whitney para os dados não-paramétricos. Os resultados foram representados em média \pm erro-padrão. A significância foi definida para $p<0,05$.

\section{RESULTADOS}

Contagens totais e diferenciais de leucócitos sanguíneos. Os dados da contagem de leucócitos no san- gue periférico demonstram que a endotoxemia aumentou o número de leucócitos totais e neutrófilos, e reduziu os valores percentuais de linfócitos. Não houve diferença nos valores de monócitos e eosinófilos (Quadro 1).

Contagens totais e diferenciais de leucócitos do LBA. A endotoxemia aumentou o número de leucócitos totais e neutrófilos no LBA e em contrapartida, houve redução nos valores percentuais de macrófagos e linfócitos. Não houve diferença entre os grupos quanto ao número de eosinófilos (Quadro 1).

Liberação de superóxido por macrófagos. No grupo endotoxêmico, houve aumento na produção de $\mathrm{O}_{2}{ }^{-}$no sobrenadante de cultura de macrófagos após $1 \mathrm{~h}$ e $2 \mathrm{~h}$ de estímulo com PMA (Fig.1).

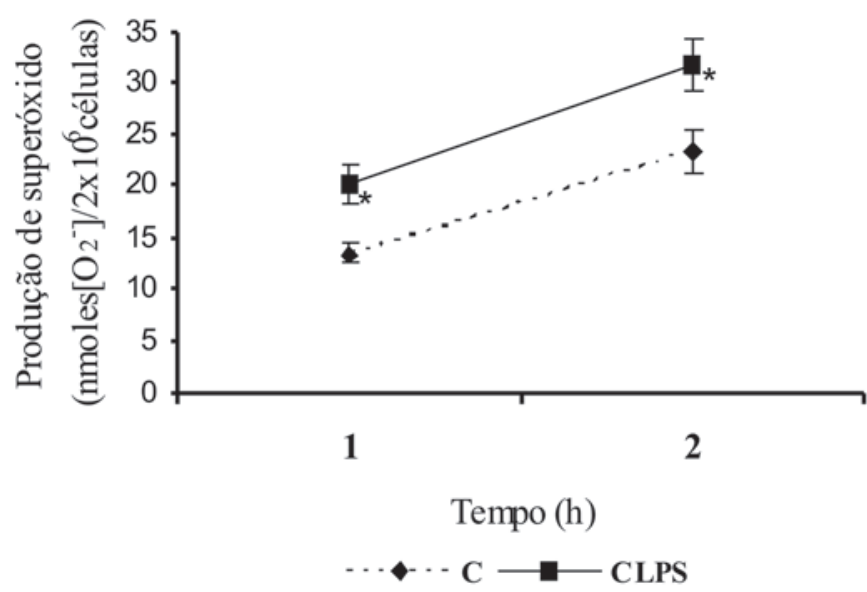

Fig.1. Produção de superóxido em sobrenadante de cultura de macrófagos estimulados com PMA nos tempos de $1 \mathrm{~h}$ e $2 \mathrm{~h}$. Os resultados são representados em média \pm erro-padrão para 12 animais. ${ }^{*} \mathrm{p}<0,05$.

Atividade da SOD, A atividade da SOD não diferiu entre os grupos nos tempos de $0,5 \mathrm{~min}, 10 \mathrm{~min}$ e $15 \mathrm{~min}$ (Fig.2).

\section{DISCUSSÃO}

No presente estudo, utilizou-se um modelo de endotoxemia com todas as avaliações realizadas após $24 \mathrm{~h}$ da administração sistêmica de LPS. Das análises realizadas

Quadro 1. Contagem de leucócitos em sangue e lavado broncoalveolar (LBA). Os resultados são representados em média \pm erro-padrão para 12 animais

\begin{tabular}{lccccc}
\hline \multirow{2}{*}{ Grupos } & \multicolumn{2}{c}{ C } & & \multicolumn{2}{c}{ CLPS } \\
\cline { 2 - 3 } \cline { 5 - 6 } & Sangue & LBA & & Sangue & LBA \\
\hline Leucócitos totais & $13,99 \pm 0,66$ & $483,33 \pm 29,72$ & & $19,77 \pm 1,58^{*}$ & $683,33 \pm 82,41^{*}$ \\
Monócitos/macrófagos & $0,83 \pm 0,08$ & $458,16 \pm 29,35$ & & $1,05 \pm 0,18$ & $551,25 \pm 70,30$ \\
& $6,46 \pm 0,56 \%$ & $94,64 \pm 0,82 \%$ & & $4,92 \pm 0,48 \%$ & $80,68 \pm 2,67 \%^{*}$ \\
Linfócitos & $9,67 \pm 0,59$ & $13 \pm 2,36$ & & $9,30 \pm 1,45$ & $12,16 \pm 3,52$ \\
& $69,46 \pm 2,17 \%$ & $2,92 \pm 0,51 \%$ & & $45,07 \pm 4,89 \%^{*}$ & $1,25 \pm 0,26 \% \%^{*}$ \\
Eosinófilos & $0,51 \pm 0,06$ & $2,91 \pm 1,58$ & & $0,49 \pm 0,09$ & $5,25 \pm 2,64$ \\
& $3,92 \pm 0,47 \%$ & $0,57 \pm 0,27 \%$ & & $2,61 \pm 0,59 \%$ & $0,62 \pm 0,23 \%$ \\
Neutrófilos & $2,89 \pm 0,26$ & $9,25 \pm 2,21$ & & $8,75 \pm 1,14^{*}$ & $114,66 \pm 18,92^{*}$ \\
& $20 \pm 1,91 \%$ & $1,85 \pm 0,41 \%$ & & $47,38 \pm 4,93 \% *$ & $17,43 \pm 2,70 \% *$
\end{tabular}

$$
{ }^{*} \mathrm{p}<0,05
$$




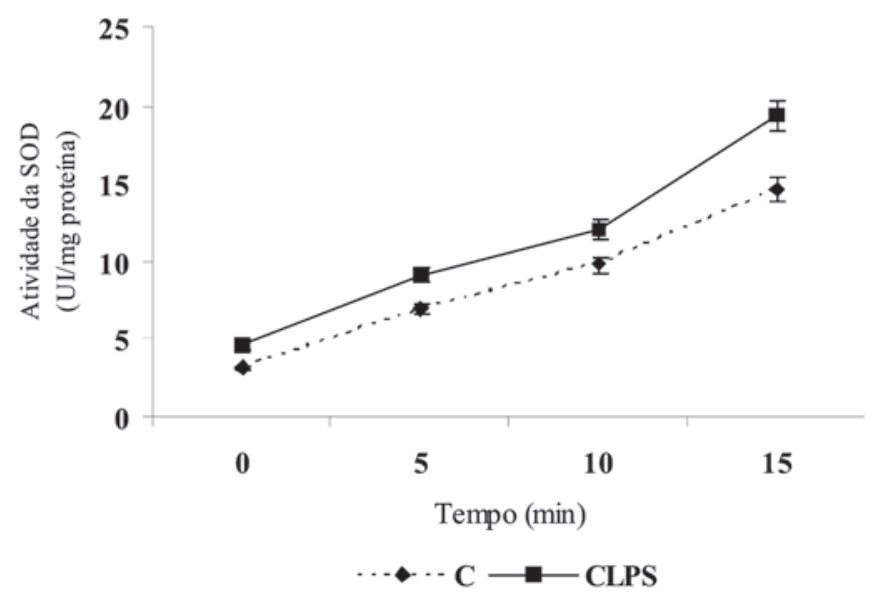

Fig.2. Atividade da superóxido dismutase (SOD) em sobrenadante de cultura de macrófagos nos tempos de $0,5 \mathrm{~min}, 10 \mathrm{~min}$ e $15 \mathrm{~min}$. Os resultados são representados em média \pm erro-padrão para 12 animais $(p>0,05)$.

nos animais endotoxêmicos pode-se constatar que houve recrutamento de neutrófilos para o pulmão e aumento na produção de superóxido pelos macrófagos alveolares. A endotoxemia não alterou a produção de superóxido dismutase em ratos Wistar.

A presença da resposta inflamatória, localizada principalmente nos pulmões, foi demonstrada pela primeira vez por Massoudy et al. (2001). O influxo leucocitário foi descrito em diversas condições pulmonares inflamatórias (Boutten et al. 1996). Neste estudo, o número de leucócitos totais e neutrófilos, em sangue periférico e LBA, aumentou após administração sistêmica de LPS.

Friedman et al. (s.d.) afirmaram que a leucocitose não é marcador específico ou sensível de infecção. Contudo, assim como mudanças na temperatura corporal, esses parâmetros são facilmente mensuráveis e continuam fundamentais na monitorização da sepse (Friedman et al. s.d., Kreimer et al. 2005).

Estudos recentes sugerem que células fagocíticas, incluindo macrófagos e neutrófilos, contribuem na fisiopatologia de injúria pulmonar induzida por endotoxina (Williams et al. 1993, Sunil et al. 2002). Uma vez que os macrófagos estão normalmente presentes nos tecidos, a maioria dos neutrófilos em resposta à endotoxina migra do sangue para o pulmão (Sunil et al. 2002). Os resultados do presente estudo estão de acordo com Bowler et al. (2004), que demonstraram aumento de neutrófilos em LBA tanto em valores absolutos quanto relativos. Estudo realizado por Sunil et al. (2002) demonstrou que o número de neutrófilos aderentes à vasculatura pulmonar aumentou na endotoxemia aguda em ratos da linhagem Wistar. Outros estudos (Haslett et al. 1987, Keubler et al. 2000) realizados em coelhos também comprovaram esses achados.

Neste trabalho, as contagens totais e diferenciais dos leucócitos em sangue periférico e LBA foram efetivas para demonstrar de forma indireta a migração destas células para o sítio inflamatório/infeccioso. Além de induzir o re- crutamento de neutrófilos para o pulmão, a endotoxemia aumentou a atividade oxidante de MA através da produção do microbicida $\mathrm{O}_{2}^{-}$. A produção desse agente oxidante é importante para a destruição dos microrganismos, porém sua produção excessiva pode causar danos às células do hospedeiro contribuindo na patogênese da injúria pulmonar (Andrade Jr et al. 2005).

A avaliação da síntese de substâncias oxidantes é uma forma sensível e conveniente de se monitorar a função dos macrófagos (Mayo \& Curnutte 1990). Estudo demonstrou aumento na produção de $\mathrm{O}_{2}^{-}$e óxido nítrico em artéria pulmonar de ratos endotoxêmicos (Brovkovych et al. 2001). Ainda, corroborando com os nossos resultados, alguns trabalhos revelaram aumento na produção de $\mathrm{O}_{2}^{-}$ (Wizemann \& Laskin 1994) em macrófagos no pulmão de ratos endotoxêmicos.

A atividade da enzima antioxidante SOD, neste estudo, não diferiu entre os grupos. Contudo, houve uma tendência a valores mais elevados após administração de LPS. Tal fato é relevante uma vez que estudos prévios sugerem que a SOD atenua a injúria pulmonar em vários modelos experimentais (Bowler et al. 2004). De acordo com alguns autores (Cox et al. 1995, Bowler et al. 2004), a SOD pode modular a inflamação atenuando a liberação de citocinas pró-inflamatórias por MA, que recrutam os neutrófilos para o pulmão.

Apesar da indução de resposta inflamatória pulmonar, o presente trabalho, diferentemente de outros (Bowler et al. 2003, Bowler et al. 2004), não demonstrou deficiência na atividade da SOD relacionada a este processo inflamatório. Esses resultados sugerem que neste sítio inflamatório/infeccioso, o pulmão, a SOD não modula a inflamação mesmo sem apresentar atividade reduzida.

O conhecimento dos mecanismos envolvidos na resposta inflamatória pulmonar deve ser o primeiro passo para a definição de estratégias adequadas de profilaxia e controle veterinários.

\section{CONCLUSÕES}

A endotoxemia induz a uma resposta inflamatória no pulmão de ratos Wistar representada por recrutamento de neutrófilos para este órgão e um aumento na atividade oxidante dos MA. Porém, não altera a atividade antioxidante em ratos adultos. Isto é importante para a defesa do hospedeiro contra agentes infecciosos, no entanto tal fato também pode contribuir para o surgimento de doenças pulmonares.

\section{REFERÊNCIAS}

Amersfoort E.S.V., Berkel T.J.C.V. \& Kuiper J. 2003. Receptors, mediators, and mechanisms involved in bacterial sepsis and septic shock. Clin. Microbiol. Rev. 16:379-414.

Andrade Jr D.R., Souza R.B., Santos S.A. \& Andrade D.R. 2005. Os radicais livres de oxigênio e as doenças pulmonares. J. Bras. Pneumol. 31:60-68.

Boutten A., Dehoux M.S., Seta N., Ostinelli J., Venembre P., Crestani B., Dombret M.C., Durand G. \& Aubier M. 1996. Compartmentalized IL-8 and elastase release within the human lung in unilateral pneumonia. Am. J. Respir. Crit. Care Med. 153:336-342. 
Bowler R.P., Arcaroli J., Abraham E., Patel M., Chang L.Y. \& Crapo J.D. 2003. Evidence for extracellular superoxide dismutase as a mediator of hemorrhage-induced lung injury. Am. J. Physiol. Lung Cell. Mol. Physiol. 284:680-687.

Bowler R.P., Nicks M., Tran K., Tanner G., Chang L.Y., Young S.K. \& Worthen G.S. 2004. Extracellular superoxide dismutase attenuates lipopolysaccharide-induced neutrophilic inflammation. Am. J. Respir. Cell Mol. Biol. 31:432-439.

Brovkovych V., Dobrucki L.W., Brovkovych S., Dobrucki I., Kalinowski L., Kiechle F. \& Malinski T. 2001. Nitric oxide measurements during endotoxemia. Clin. Chem. 47:1068-1074.

Cox G., Crossley J. \& Xing Z. 1995. Macrophage engulfment of apoptotic neutrophils contributes to the resolution of acute pulmonary inflammation in vivo. Am. J. Respir. Cell Mol. Biol. 12:232-237.

D'Acampora A.J., Tramonte R., Carvalho R.O.M., Ortellado D.K. \& Serafim J.D.M. 2001. Pulmão e peritônio após sepse experimental de origem abdominal em ratos Wistar. Revta Col. Bras. Cirur. 28:276.

De Castro C.M.M.B., Bureau M.F., Nahori M.A., Dumarey C.H., Vargaftig B.B. \& Bachelet M. 1995. Modulation by dexamethasone of phospholipase $A_{2}$ activities in endotoxemic guinea pigs. J. Appl. Physiol. 79:12711277.

De Castro C.M.M.B., De Castro R.M., Andrade A.D. \& Brandt C.T. 1997. LPS bacteriano: um mediador de inflamação. Anais Fac. Med. Univ. Fed. Pernambuco 42:78-83.

De Castro C.M.M.B., De Castro R.M., Medeiros A.F., Santos A.Q., Silva W.T.F. \& Filho J.L.L. 2000. Effect of stress on the production of $\mathrm{O}_{2}-$ in alveolar macrophages. J. Neuroimmunol. 108(1/2):68-72.

Dinarello C.A. 1996. Biological basis for interleukin-1 in disease. Blood 87:2065-2147.

Friedman G., Lobo S. \& Rigato O. s.d. Consenso Brasileiro de Sepse: diagnóstico, monitorização da resposta inflamatória. Disponível em: http://www.medicinaintensiva.com.br/consenso-sepse.htm. Acesso em jun 2006.

Haslett C., Worthen G.S., Giclas P.C., Morrison D.C., Henson J.E. \& Henson P.M. 1987. The pulmonary vascular sequestration of neutrophils in endotoxemia is initiated by an effect of endotoxin on the neutrophil in the rabbit. Am. Rev. Respir. Dis. 136:9-18.

Hewett J.A. \& Roth R.M. 1993. Hepatic and extraheptaic pathobiology of bacterial lipopolysaccharide. Pharmacol. Rev. 45:382-411.

Johnston Jr R.B. 1984. Measurement of $\mathrm{O}_{2}^{-}$secreted by monocytes and macrophages. Methods Enzymol. 105:365-369.
Keubler W.M., Borges J., Sckell A., Kuhnle G.E., Bergh K., Messmer K. \& Goetz A.E. 2000. Role of L-selectin in leukocyte sequestration in lung capillaries in a rabbit model of endotoxemia. Am. J. Respir. Crit. Care Med. 161:36-43.

Kreimer F., Aguiar J.L.A., Castro C.M.M.B., Lacerda C.M., Reis T. \& Júnior F.L. 2005. Resposta terapêutica e inflamatória de ratos com peritonite secundária submetidos ao uso tópico de ampicilina/sulbactam. Acta. Cirurg. Bras. 20:63-71.

Lowry O.H., Rosebrough N.J., Farr A.L. \& Randall R.J. 1951. Protein measurement with the folin phenol reagent. J. Biol. Chem. 193:265275.

Mafra D., Abdalla D.S.P. \& Cozzolino S.M.F. 1999. Peroxidação lipídica em pacientes com insuficiência renal crônica. Revta Nutr. 12:205212.

Massoudy P., Zahler S., Becker B.F., Braun S.L., Barankay A. \& Meisner H. 2001. Evidence for inflammatory responses of the lungs during coronary artery bypass grafting with cardiopulmonary bypass. Chest 119:31-36.

Mayo L.A. \& Curnutte J.T. 1990. Kinetic microplate assay for SP by neutrophils and other phagocytic cells. Methods Enzymol. 186:567575.

Meyer J., Hinder F., Stothert J. Jr, Traber L.D., Herndon D.N., Flynn J.T. \& Traber D.L. 1994. Increased organ blood flow in chronic endotoxemia is reversed by nitric oxide synthase inhibition. J. Appl. Physiol. 76:2785-2793.

Plata-Salamán C.R. 1996. Anorexia during acute and chronic disease. Nutrition 12:69-78.

Sunil V.R., Connor A.J., Zhou P., Gordon M.K., Laskin J.D. \& Laskin D.L. 2002. Activation of adherent vascular neutrophils in the lung during acute endotoxemia. Respir. Res. 3:21.

Wang C.Z., Herndon D.N., Traber L.D., Yang S.F., Cox R.A., Nakazawa H., Barrow R.E. \& Traber D.L. 1994. Pulmonary inflammatory cell response to sustained endotoxin administration. J. Appl. Physiol. 76:516-522.

Williams J.H. Jr, Patel S.K., Hatakeyama D., Arian R., Guo K., Hickey T.J., Liao S.Y. \& Ulich T.R. 1993. Activated pulmonary vascular neutrophils as early mediators of endotoxin-induced lung inflammation. Am. J. Respir. Cell Mol. Biol. 8:134-144.

Wizemann T.M. \& Laskin D.L. 1994. Enhanced phagocytosis, chemotaxis, and production of reactive oxygen intermediates by interstitial lung macrophages following acute endotoxemia. Am. J. Respir. Cell Mol. Biol. 11:358-365. 